\title{
The Probability that a Random Probable Prime is Composite*
}

\author{
By Su Hee Kim and Carl Pomerance**
}

\begin{abstract}
Consider a procedure which (1) chooses a random odd number $n \leq x,(2)$ chooses a random number $b, 1<b<n-1$, and (3) accepts $n$ if $b^{n-1} \equiv 1(\bmod n)$. Let $P(x)$ denote the probability that this procedure accepts a composite number. It is known from work of Erdös and the second author that $P(x) \rightarrow 0$ as $x \rightarrow \infty$. In this paper, explicit inequalities are established for $P(x)^{\circ}$. For example, it is shown that $P\left(10^{100}\right)<2.77 \times 10^{-8}$ and that $P(x) \leq(\log x)^{-197}$ for $x \geq 10^{10^{5}}$.
\end{abstract}

Introduction. Suppose one wants to produce a random prime $p \leq x$, drawn with the uniform distribution. One possible solution is to choose a random number $n, 1<n \leq x$, and apply a test to $n$ that can tell if it is prime or composite. This procedure is repeated independently until a prime is found. By the prime number theorem, the expected number of trials until a prime is drawn is about $\log x$. If one wishes to choose an odd prime, the trials $n$ may be restricted to odd numbers. The expected number of trials is then about $\frac{1}{2} \log x$.

There are many algorithms which can be used to decide if $n$ is prime or composite. However, using the Fermat congruence is a very cheap test that is usually recommended as a preliminary procedure before a more time-consuming test is attempted. Namely, one chooses a random number $b, 1<b<n-1$, and checks if $b^{n-1} \equiv 1(\bmod n)$. If $n$ is prime, then this congruence will hold. If this congruence holds, then $n$ is called a probable prime to the base $b$. This procedure can prove an input $n$ is composite, but cannot establish primality.

How good is this test at producing random primes? Specifically, let $P(x)$ denote the probability that $n$ is composite given that

(i) $n$ is chosen at random with $1<n \leq x, n$ odd,

(ii) $b$ is chosen at random with $1<b<n-1$, and

(iii) $n$ is a probable prime to the base $b$.

It is well known that there are some composite numbers $n$, namely the Carmichael numbers, such that (iii) holds for every $b$ coprime to $n$. However, Carmichael numbers are rare, so presumably the odds of choosing one in (i) is small. In fact, extensive numerical evidence suggests that $P(x)$ is quite small when $x$ is large.

In practice, if a large random number $n$ passes a random probable prime test, then one strongly conjectures that $n$ is prime. As Henri Cohen has colorfully put it, such an $n$ can be considered an "industrial grade prime." That is, although $n$

Received November 8, 1988.

1980 Mathematics Subject Classification (1985 Revision). Primary 11Y11; Secondary 11A51, 11 N56.

*This paper is based on the first author's master's thesis at the University of Georgia.

** Supported in part by an NSF grant. 
has not been proved prime, the probability it is composite is so small that $n$ might be used as a prime for industrial (cryptographic) purposes.

We do know theoretically that if $x$ is sufficiently large, then $P(x)$ is small. Indeed, from Theorem 2.2 in Erdös and Pomerance [2], we have that

$$
P(x) \leq \exp (-(1+o(1)) \log x \log \log \log x / \log \log x)
$$

as $x \rightarrow \infty$. In particular, $\lim P(x)=0$.

Although we have the strong inequality (1.1) and the practical experience of many people to draw on, we still do not have any good estimate for $P(x)$ for various finite values of $x$. The problem is the " $o(1)$ " in (1.1) which renders the inequality computationally useless.

In this paper we replace the asymptotic inequality (1.1) with a weaker, but explicit inequality. The argument is loosely based on the proof in [2] of (1.1) above, but a number of difficulties are encountered. For delicate estimates involving prime numbers, we use the results of Rosser and Schoenfeld [5]. However, the rest of our work is elementary and involves only moderate computation.

We prove that

$$
P(x) \leq(\log x)^{-197} \text { for } x \geq 10^{10^{5}} .
$$

For smaller values of $x$, our results are summarized in Table 1. To find an upper estimate for $P(x)$ for some $x$ not in the table with $10^{60}<x<10^{10^{5}}$, one can find the largest $x_{0}$ in the table with $x_{0}<x$ and multiply the estimate at $x_{0}$ by $\log x / \log x_{0}$.

It is highly likely that our upper bounds can be improved upon. To some extent, it is a matter of how hard one is willing to work. Sometimes we make trivial estimates for simplicity, but a more careful estimation would give a better result.

One possible way to gain an improvement is to replace the Fermat congruence with the strong probable prime test of Selfridge. This test is just as easy to perform and it "lies" less frequently about composite numbers. To describe this test, let $n>1$ be an odd number. First one computes $s, t$ with $n-1=2^{s} t$ and $t$ odd. Next, one chooses a number $b, 1<b<n-1$. The number $n$ passes the test (and is called a strong probable prime to the base $b$ ) if either

$$
b^{t} \equiv 1(\bmod n) \text { or } \quad b^{2^{i} t} \equiv-1(\bmod n) \text { for some } i<s .
$$

Every odd prime must pass this test. Moreover, Monier [3] and Rabin [4] have shown that if $n>1$ is an odd composite, then the probability that it is a strong probable prime to a random base $b, 1<b<n-1$, is less than $\frac{1}{4}$.

Let $P_{1}(x)$ denote the same probability as $P(x)$, except that (iii) is changed to

(iii)' $n$ is a strong probable prime to the base $b$.

Based on the Monier-Rabin theorem, one is tempted to say that $P_{1}(x) \leq \frac{1}{4}$, but as pointed out in [1], this reasoning is fallacious. In fact, if $\alpha$ is the probability that a random odd number up to $x$ is prime and $\beta$ is an upper bound for the probability that an odd composite number up to $x$ passes a random strong probable prime test, then

$$
P_{1}(x) \leq \frac{(1-\alpha) \beta}{\alpha+(1-\alpha) \beta}
$$




\section{TABLE $1^{* * *}$}

$\begin{array}{cccc}x & \begin{array}{c}\text { Upper bound } \\ \text { for } P(x)\end{array} & x & \begin{array}{c}\text { Upper bound } \\ \text { for } P(x)\end{array} \\ 1.0 \mathrm{E}+60 & 7.16 \mathrm{E}-2 & 1.0 \mathrm{E}+0300 & 5.8 \mathrm{E}-0029 \\ 1.0 \mathrm{E}+70 & 2.87 \mathrm{E}-3 & 1.0 \mathrm{E}+0400 & 5.7 \mathrm{E}-0042 \\ 1.0 \mathrm{E}+80 & 8.46 \mathrm{E}-5 & 1.0 \mathrm{E}+0500 & 2.3 \mathrm{E}-0055 \\ 1.0 \mathrm{E}+90 & 1.70 \mathrm{E}-6 & 1.0 \mathrm{E}+0600 & 1.7 \mathrm{E}-0068 \\ 1.0 \mathrm{E}+100 & 2.77 \mathrm{E}-8 & 1.0 \mathrm{E}+0700 & 1.8 \mathrm{E}-0082 \\ 1.0 \mathrm{E}+110 & 4.03 \mathrm{E}-10 & 1.0 \mathrm{E}+0800 & 5.4 \mathrm{E}-0096 \\ 1.0 \mathrm{E}+120 & 5.28 \mathrm{E}-12 & 1.0 \mathrm{E}+0900 & 1.0 \mathrm{E}-0109 \\ 1.0 \mathrm{E}+130 & 7.54 \mathrm{E}-14 & 1.0 \mathrm{E}+1000 & 1.2 \mathrm{E}-0123 \\ 1.0 \mathrm{E}+140 & 1.08 \mathrm{E}-15 & 1.0 \mathrm{E}+2000 & 8.6 \mathrm{E}-0262 \\ 1.0 \mathrm{E}+150 & 1.49 \mathrm{E}-17 & 1.0 \mathrm{E}+3000 & 3.8 \mathrm{E}-0397 \\ 1.0 \mathrm{E}+160 & 1.81 \mathrm{E}-19 & 1.0 \mathrm{E}+4000 & 7.8 \mathrm{E}-0537 \\ 1.0 \mathrm{E}+170 & 2.27 \mathrm{E}-21 & 1.0 \mathrm{E}+5000 & 7.6 \mathrm{E}-0680 \\ 1.0 \mathrm{E}+180 & 2.76 \mathrm{E}-23 & 1.0 \mathrm{E}+6000 & 3.9 \mathrm{E}-0820 \\ 1.0 \mathrm{E}+190 & 3.26 \mathrm{E}-25 & 1.0 \mathrm{E}+7000 & 1.1 \mathrm{E}-0951 \\ 1.0 \mathrm{E}+200 & 3.85 \mathrm{E}-27 & 1.0 \mathrm{E}+8000 & 7.3 \mathrm{E}-1081 \\ & & 1.0 \mathrm{E}+9000 & 1.7 \mathrm{E}-1207 \\ & & 1.0 \mathrm{E}+10000 & 1.6 \mathrm{E}-1331 \\ & & 1.0 \mathrm{E}+100000 & 1.3 \mathrm{E}-10584\end{array}$

From Monier-Rabin, we have that $\beta \leq \frac{1}{4}$. Thus all we get from this theorem is that

$$
P_{1}(x) \leq \frac{1-\alpha}{1+3 \alpha}
$$

If $x$ is very large, then $\alpha$ is very small and so (1.5) is a quite weak result.

However, presumably much is lost using the worst case upper bound $\beta$. This is attained only for very special composites which, like Carmichael numbers, are rare.

The results of this paper also apply to $P_{1}(x)$, since we trivially have $P_{1}(x) \leq$ $P(x)$. If one were to concentrate solely on $P_{1}(x)$, it is possible that considerably stronger estimates could be obtained. We remark that by using the formulas of Monier [3] for the number of $b$ for which $n$ is a probable prime, respectively strong probable prime, our estimates for $P(x)$ can be multiplied by $\frac{1}{2}$ when applied to $P_{1}(x)$.

Consider finally a procedure which chooses a random odd number $n \leq x$ and then performs $k$ strong probable prime tests on $n$ with $k$ independently drawn random numbers $b, 1<b<n-1$. Let $P_{k}(x)$ denote the probability that this

\footnotetext{
${ }^{* * *}$ The notation $a E n$ means $a \times 10^{n}$.
} 
procedure accepts a composite number. Combining our results with the MonierRabin theorem, we have

$$
P_{k}(x) \leq 4^{-(k-1)} P_{1}(x) /\left(1-P_{1}(x)\right) \leq 4^{-(k-1)} P(x) /(1-P(x)) .
$$

The popularly believed inequality is that $P_{k}(x) \leq 4^{-k}$, but as we have seen, the reasoning for this is fallacious. However, if we have $P(x) \leq \frac{1}{5}$, then (1.6) does imply that $P_{k}(x) \leq 4^{-k}$ for every $k$. In particular, from the results of this paper, this inequality holds for all $x \geq 10^{60}$.

2. The Basic Method. Let

$$
F(n)=\#\left\{b \in(\mathbf{Z} / n)^{*}: b^{n-1} \equiv 1(\bmod n)\right\} .
$$

If $n>1$ is odd, then $b= \pm 1$ both satisfy $b^{n-1} \equiv 1(\bmod n)$. Thus for these $n$, $F(n)-2$ is the number of $b, 1<b<n-1$, with $b^{n-1} \equiv 1(\bmod n)$. Also note that by Fermat's theorem, if $p$ is a prime, then $F(p)=p-1$. We thus have for $x \geq 5$,

$$
\begin{aligned}
P(x) & =\frac{\sum_{n \leq x, n \text { odd, composite }}(F(n)-2)}{\sum_{1<n \leq x, n \text { odd }}(F(n)-2)} \\
& \leq \frac{\sum_{n \leq x, n \text { odd, composite }} F(n)}{\sum_{2<p \leq x}(p-3)},
\end{aligned}
$$

where here and throughout the paper, $p$ denotes a prime.

Hence, to get an upper bound for $P(x)$, we shall be interested in obtaining a lower bound for $\sum_{2<p \leq x}(p-3)$ and an upper bound for $\sum_{n \leq x, n \text { odd, composite }} F(n)$.

For this purpose we shall prove two theorems.

THEOREM 2.1. For $x \geq 37$, we have

$$
\sum_{2<p \leq x}(p-3) \geq \frac{x^{2}}{2(2+\log x)} .
$$

THEOREM 2.2. Suppose $c, L_{1}$ and $L$ are arbitrary real numbers satisfying $\frac{1}{2}<$ $c<1,1<L_{1}<L$. Then for any $x>L^{2}$, we have

$$
\begin{aligned}
& \sum_{\substack{n \leq x, n \text { odd, } \\
\text { composite }}} F(n) \\
& \leq \frac{x^{2}}{4 L_{1}}+\frac{x^{2}}{L}\left(1+\log L_{1}\right)+\frac{x^{2}}{2(L-1)} \cdot\left(\frac{L_{1}}{L-1}+1\right)\left(2+\log L_{1}\right)^{2} \\
& \quad+\frac{K_{c}}{1-c} x^{1+c} L^{2(1-c)}\left(1+\log L_{1}\right) \sum_{m \leq L^{2} L_{1}} \frac{\tau_{L_{1}}(m)}{m} \exp \left(2^{-c} f_{c}(m)\right),
\end{aligned}
$$

where

$$
\begin{gathered}
K_{c}=\exp \left(\sum_{p>2} \sum_{k=2}^{\infty} k^{-1} p^{-k c}\right), \\
f_{c}(m)=\prod_{p \mid m}\left(1-p^{-c}\right)^{-1},
\end{gathered}
$$

and $\tau_{L_{1}}(m)$ is the number of divisors of $m$ up to $L_{1}$.

Before we prove Theorems 2.1 and 2.2, we state a theorem that is an immediate consequence of them and (2.1). Say that $g\left(x, c, L, L_{1}\right)$ is the right member of the inequality in Theorem 2.2 . 
THEOREM 2.3. For all real numbers $c, L$ and $L_{1}$ with $\frac{1}{2}<c<1,1<L_{1}<L$ and for all $x>L^{2}>37$, we have

$$
P(x) \leq 2(2+\log x) g\left(x, c, L, L_{1}\right) / x^{2} .
$$

Thus, our upper bound for $P(x)$ depends on the choices of the variables $x, c, L$ and $L_{1}$.

Now let us prove Theorems 2.1 and 2.2.

Proof of Theorem 2.1. Let $\pi(x)$ denote the number of primes not exceeding $x$. We have

$$
\pi(x)>x /\left(\log x-\frac{1}{2}\right) \quad \text { for } x \geq 67
$$

and

$$
\pi(x)<x /\left(\log x-\frac{3}{2}\right) \quad \text { for } x>e^{3 / 2}(x \geq 4.48169)
$$

by (3.3) and (3.4) in [5, p. 69].

Using partial summation, (2.2) and (2.3), we have

$$
\begin{aligned}
\sum_{2<p \leq x}(p-3) & =(x-3) \pi(x)-\int_{3}^{x} \pi(t) d t \\
& >\frac{x(x-3)}{\log x-\frac{1}{2}}-\int_{3}^{x} \pi(t) d t .
\end{aligned}
$$

Now for $x \geq 245$,

$$
\begin{aligned}
\int_{3}^{x} \pi(t) d t \leq & \int_{245}^{x} \frac{t d t}{\log t-\frac{3}{2}}+\int_{3}^{245} \pi(t) d t \\
= & \frac{x^{2}}{2\left(\log x-\frac{3}{2}\right)}-\frac{245^{2}}{2\left(\log 245-\frac{3}{2}\right)} \\
& +\frac{1}{2} \int_{245}^{x} t\left(\log t-\frac{3}{2}\right)^{-2} d t+7154 \\
\leq & \frac{x^{2}}{2\left(\log x-\frac{3}{2}\right)}+\frac{1}{2} \int_{245}^{x} t\left(\log t-\frac{3}{2}\right)^{-2} d t
\end{aligned}
$$

Let $S=\frac{1}{2} \int_{245}^{x} t\left(\log t-\frac{3}{2}\right)^{-2} d t$. We have

$$
\begin{aligned}
S & <\frac{1}{4} x^{2}\left(\log x-\frac{3}{2}\right)^{-2}+\frac{1}{2} \int_{245}^{x} t\left(\log t-\frac{3}{2}\right)^{-3} d t \\
& <\frac{1}{4} x^{2}\left(\log x-\frac{3}{2}\right)^{-2}+\frac{1}{4} S
\end{aligned}
$$

because $\log t-\frac{3}{2}>4$ for $t \geq 245$. Therefore,

$$
S<\frac{1}{3} x^{2}\left(\log x-\frac{3}{2}\right)^{-2},
$$

so that

$$
\int_{3}^{x} \pi(t) d t<\frac{x^{2}}{2\left(\log x-\frac{3}{2}\right)}+\frac{x^{2}}{3\left(\log x-\frac{3}{2}\right)^{2}} .
$$

Putting this estimate in (2.4), we have for $x \geq 245$

$$
\sum_{2<p \leq x}(p-3) \geq \frac{x(x-3)}{\log x-\frac{1}{2}}-\frac{x^{2}}{2\left(\log x-\frac{3}{2}\right)}-\frac{x^{2}}{3\left(\log x-\frac{3}{2}\right)^{2}} .
$$


We replace the right side of (2.5) with the simpler expression $x^{2} / 2(2+\log x)$ which is smaller for all $x>20,000$. Moreover, we have checked numerically that

$$
\sum_{2<p \leq n-1}(p-3)>\frac{n^{2}}{2(2+\log n)}
$$

for every integer $n$ with $38 \leq n \leq 20,000$. Thus we have Theorem 2.1.

Proof of Theorem 2.2. Since $F(n)$ is the cardinality of a subgroup of $(\mathbf{Z} / n)^{*}$, we have that for any $n, F(n) \mid \phi(n)$, where $\phi$ is Euler's function. That is, $F(n)=\phi(n) / k$ for some integer $k>0$.

Let $\mathbf{C}_{k}(x)$ denote the set of odd, composite $n \leq x$ such that $F(n)=\phi(n) / k$, and let $C_{k}(x)=\# \mathrm{C}_{k}(x)$.

For any $x>L^{2}$ where $L>L_{1}>1$,

$$
\begin{aligned}
\sum_{\substack{n \leq x, n \text { odd, } \\
\text { composite }}} F(n) & =\sum_{k=1}^{\infty} \sum_{n \in \mathbf{C}_{k}(x)} F(n)=\sum_{k=1}^{\infty} \sum_{n \in \mathbf{C}_{k}(x)} \frac{\phi(n)}{k} \\
& =\sum_{k \leq L_{1}} \frac{1}{k} \sum_{n \in \mathbf{C}_{k}(x)} \phi(n)+\sum_{k>L_{1}} \sum_{n \in \mathbf{C}_{k}(x)} \frac{\phi(n)}{k} \\
& \leq x \sum_{k \leq L_{1}} \frac{C_{k}(x)}{k}+\frac{1}{L_{1}} \sum_{\substack{1<n \leq x \\
n \text { odd }}}(n-2) \\
& \leq x \sum_{k \leq L_{1}} \frac{C_{k}(x)}{k}+\frac{x^{2}}{4 L_{1}} .
\end{aligned}
$$

It will thus be desirable to obtain an upper bound for $C_{k}(x)$. Three classes are considered to estimate $C_{k}(x)$ for $k \leq L_{1}$ :

(i) $n \leq x / L$,

(ii) $n$ is divisible by some prime $p>L$,

(iii) $n>x / L$ and every prime $p$ in $n$ is at most $L$.

Let $C_{k, 1}(x), C_{k, 2}(x)$ and $C_{k, 3}(x)$ denote the number of $n \leq x$ counted by $C_{k}(x)$ for each class respectively. Thus,

$$
C_{k}(x) \leq C_{k, 1}(x)+C_{k, 2}(x)+C_{k, 3}(x)
$$

Obviously,

$$
C_{k, 1}(x) \leq x / 2 L
$$

We now state a result that will be useful for classes (ii) and (iii). This result is (2.11) in [2].

LEMMA 2.4. If $F(n)=\phi(n) / k$, then $\lambda(n) \mid k(n-1)$, where $\lambda(n)$ is the Carmichael universal exponent function; that is, $\lambda(n)$ is the least positive integer with $b^{\lambda(n)} \equiv 1(\bmod n)$ for all integers $b$ with $(b, n)=1$.

Let $d$ be a natural number. We consider those $n$ counted by $C_{k}(x)$ with $d \mid n$. If $d \mid n$, then $\lambda(d) \mid \lambda(n)$, so that the condition $\lambda(n) \mid k(n-1)$ from Lemma 2.4 implies $\lambda(d) \mid k(n-1)$. Thus, the number of $n$ counted by $C_{k}(x)$ with $d \mid n$ is at most the number of composite numbers $n \leq x$ with

$$
n \equiv 0 \quad(\bmod d), \quad k(n-1) \equiv 0(\bmod \lambda(d)) .
$$


The latter congruence is equivalent to

$$
n-1 \equiv 0\left(\bmod \frac{\lambda(d)}{(k, \lambda(d))}\right) .
$$

If there is any such $n$ that satisfies (2.9), it is necessary that

$$
\left(d, \frac{\lambda(d)}{(k, \lambda(d))}\right)=1
$$

Thus, by the Chinese remainder theorem, the number of $n$ counted by $C_{k}(x)$ with $d \mid n$ is at most

$$
1+\left[\frac{x(k, \lambda(d))}{d \lambda(d)}\right] .
$$

Further, if $d=p$ is prime, then the solution $n=p$ of (2.9) should not be counted since it is not composite. Thus for $p$ prime, the number of $n$ counted by $C_{k}(x)$ with $p \mid n$ is at most

$$
\left[\frac{x(k, p-1)}{p(p-1)}\right] \text {. }
$$

We now estimate $C_{k, 2}(x)$ by using (2.11). For any $k \leq L_{1}<L$,

$$
\begin{aligned}
C_{k, 2}(x) & \leq x \sum_{p>L} \frac{(k, p-1)}{p(p-1)}=x \sum_{d \mid k} \sum_{\substack{p>L \\
(k, p-1)=d}} \frac{d}{p(p-1)} \\
& \leq x \sum_{d \mid k} \sum_{\substack{p>L \\
p-1=m d \\
\text { for some } m}} \frac{d}{(m d+1) m d}<x \sum_{d \mid k} \sum_{m>(L-1) / d} \frac{1}{m^{2} d} \\
& \leq x \sum_{d \mid k} \frac{1}{d}\left(\frac{d^{2}}{(L-1)^{2}}+\int_{(L-1) / d}^{\infty} \frac{1}{t^{2}} d t\right) \\
& =x \sum_{d \mid k}\left(\frac{d}{(L-1)^{2}}+\frac{1}{(L-1)}\right) \leq \frac{x}{L-1}\left(\frac{L_{1}}{L-1}+1\right) \tau(k),
\end{aligned}
$$

where $\tau(k)$ is the number of divisors of $k$.

Suppose $n$ is in class (iii). Let $d_{0}$ be the least divisor of $n$ with $d_{0}>x / L^{2}$. If $p$ is any prime factor of $d_{0}$ and $d_{0}>x / L$, then $d_{0} / p \geq d_{0} / L>x / L^{2}$, which gives a contradiction. Hence, $n$ must have a divisor $d$ with

$$
\frac{x}{L^{2}}<d \leq \frac{x}{L}
$$

Thus by $(2.10)$,

$$
C_{k, 3}(x) \leq \sum^{\prime}\left(1+\left[\frac{x(k, \lambda(d))}{d \lambda(d)}\right]\right)
$$

where $\sum^{\prime}$ denotes a sum over odd $d$ satisfying (2.13). 
We thus have

$$
\begin{aligned}
C_{k, 3}(x) & \leq \frac{x}{2 L}+x \sum_{d \lambda(d) \leq x(k, \lambda(d))}^{\prime} \frac{(k, \lambda(d))}{d \lambda(d)} \\
& =\frac{x}{2 L}+x \sum_{m \leq L^{2}} \frac{1}{m} \sum_{\lambda(d) /(k, \lambda(d))=m}^{\prime} \frac{1}{d} \\
& =\frac{x}{2 L}+x \sum_{u \mid k} \sum_{m \leq L^{2}} \frac{1}{m} \sum_{\substack{\lambda(d)=m u \\
(k, \lambda(d))=u}}^{\prime} \frac{1}{d} \\
& \leq \frac{x}{2 L}+x \sum_{u \mid k} \sum_{m \leq L^{2}} \frac{1}{m} \sum_{\lambda(d)=m u}^{\prime} \frac{1}{d} .
\end{aligned}
$$

Using partial summation for the inner sum in (2.15), we have

$$
\sum_{\lambda(d)=m u}^{\prime} \frac{1}{d}=\frac{1}{x / L} \sum_{\lambda(d)=m u}^{\prime} 1+\int_{x / L^{2}}^{x / L} \frac{1}{t^{2}} \sum_{\substack{d \leq t \\ \lambda(d)=m u}}^{\prime} 1 d t .
$$

We thus shall be interested in obtaining an upper bound for $\Lambda(t, m u)$, the number of odd $d \leq t$ with $\lambda(d)=m u$.

LEMMA 2.5. Let $\lambda(m)$ be the Carmichael universal exponent function. Then,

$$
\begin{aligned}
\Lambda(x, n) & :=\#\{m \leq x: m \text { odd }, \lambda(m)=n\} \\
& \leq K_{c} x^{c} \exp \left(2^{-c} f_{c}(n)\right)
\end{aligned}
$$

for any $x>1, \frac{1}{2}<c<1$, where $K_{c}$ and $f_{c}(n)$ are defined in Theorem 2.2.

The proof of Lemma 2.5 will be given later. Using it now in (2.16), we have

$$
\begin{aligned}
\sum_{\lambda(d)=m u}^{\prime} \frac{1}{d} \leq & \frac{L}{x}\left(\frac{x}{L}\right)^{c} K_{c} \exp \left(2^{-c} f_{c}(m u)\right) \\
& +\int_{x / L^{2}}^{x / L} \frac{1}{t^{2}} t^{c} K_{c} \exp \left(2^{-c} f_{c}(m u)\right) d t \\
= & \left(\frac{L}{x}\right)^{1-c} K_{c} \exp \left(2^{-c} f_{c}(m u)\right) \\
& +K_{c} \exp \left(2^{-c} f_{c}(m u)\right) \cdot \frac{1}{c-1} \cdot\left[\left(\frac{x}{L}\right)^{c-1}-\left(\frac{x}{L^{2}}\right)^{c-1}\right] \\
\leq & \frac{K_{c}}{1-c}\left(\frac{L^{2}}{x}\right)^{1-c} \exp \left(2^{-c} f_{c}(m u)\right) .
\end{aligned}
$$

Putting this estimate in (2.15), we have

$$
\begin{aligned}
C_{k, 3}(x) & \leq \frac{x}{2 L}+x \sum_{u \mid k} \sum_{m \leq L^{2}} \frac{1}{m} \cdot \frac{K_{c}}{1-c}\left(\frac{L^{2}}{x}\right)^{1-c} \exp \left(2^{-c} f_{c}(m u)\right) \\
& \leq \frac{x}{2 L}+\frac{K_{c}}{1-c} x^{c} L^{2(1-c)} \sum_{u \mid k} \sum_{m \leq L^{2}} \frac{1}{m} \exp \left(2^{-c} f_{c}(m u)\right)
\end{aligned}
$$


Using estimates (2.8), (2.12) and (2.18) in (2.7), we have

$$
\begin{aligned}
C_{k}(x) \leq & \frac{x}{L}+\frac{x}{L-1}\left(\frac{L_{1}}{L-1}+1\right) \tau(k) \\
& +\frac{K_{c}}{1-c} x^{c} L^{2(1-c)} \sum_{u \mid k} \sum_{m \leq L^{2}} \frac{1}{m} \exp \left(2^{-c} f_{c}(m u)\right) .
\end{aligned}
$$

Using this estimate in (2.6), we get

$$
\begin{aligned}
& \sum_{\substack{n \leq x, n \text { odd, } \\
\text { composite }}} F(n) \\
& \leq \frac{x^{2}}{4 L_{1}}+\frac{x^{2}}{L} \sum_{k \leq L_{1}} \frac{1}{k}+\frac{x^{2}}{L-1}\left(\frac{L_{1}}{L-1}+1\right) \sum_{k \leq L_{1}} \frac{\tau(k)}{k} \\
& \quad+\frac{K_{c}}{1-c} x^{1+c} L^{2(1-c)} \sum_{k \leq L_{1}} \frac{1}{k} \sum_{u \mid k} \sum_{m \leq L^{2}} \frac{1}{m} \exp \left(2^{-c} f_{c}(m u)\right) .
\end{aligned}
$$

The single sums on the right of (2.19) are dealt with in the following lemma.

LEMMA 2.6. For any $x \geq 1$, we have

$$
\sum_{k \leq x} \frac{1}{k} \leq 1+\log x, \quad \sum_{k \leq x} \frac{\tau(k)}{k}<\frac{1}{2}(2+\log x)^{2},
$$

where $\tau(k)$ is the number of divisors of $k$.

We defer the proof of Lemma 2.6 until later.

We deal with the final triple sum on the right of (2.19) as follows. We have, using Lemma 2.6,

$$
\begin{aligned}
\sum_{k \leq L_{1}} & \frac{1}{k} \sum_{u \mid k} \sum_{m \leq L^{2}} \frac{1}{m} \exp \left(2^{-c} f_{c}(m u)\right) \\
& =\sum_{u \leq L_{1}} \sum_{m \leq L^{2}} \sum_{v \leq L_{1} / u} \frac{1}{m u v} \exp \left(2^{-c} f_{c}(m u)\right) \\
& \leq\left(1+\log L_{1}\right) \sum_{u \leq L_{1}} \sum_{m \leq L^{2}} \frac{1}{m u} \exp \left(2^{-c} f_{c}(m u)\right) \\
& \leq\left(1+\log L_{1}\right) \sum_{\mu \leq L^{2} L_{1}} \frac{\tau_{L_{1}}(\mu)}{\mu} \exp \left(2^{-c} f_{c}(\mu)\right)
\end{aligned}
$$

where $\tau_{L_{1}}(\mu)$ is the number of divisors of $\mu$ up to $L_{1}$. Using this estimate and Lemma 2.6 on the right of (2.19) immediately gives the theorem.

We now prove Lemmas 2.5 and 2.6.

Proof of Lemma 2.5. If $c>0$,

$$
\begin{aligned}
\Lambda(x, n) & =\sum_{\substack{m \leq x \\
m \text { odd } \\
\lambda(m)=n}} 1 \leq x^{c} \sum_{\substack{\lambda(m)=n \\
m \text { odd }}} m^{-c} \leq x^{c} \sum_{\substack{p|m \\
\Rightarrow(p-1)| n, p \text { odd }}} m^{-c} \\
& =x^{c} \prod_{\substack{(p-1) \mid n \\
p \text { odd }}}\left(1-p^{-c}\right)^{-1} .
\end{aligned}
$$


Hence,

$$
\begin{aligned}
\Lambda(x, n) \leq x^{c} \exp \left(-\sum_{\substack{(p-1) \mid n \\
p \text { odd }}} \log \left(1-p^{-c}\right)\right) \\
=x^{c} \exp \left(\sum_{\substack{(p-1) \mid n \\
p \text { odd }}}\left(p^{-c}+\frac{1}{2} p^{-2 c}+\frac{1}{3} p^{-3 c}+\cdots\right)\right) .
\end{aligned}
$$

We have

$$
\begin{aligned}
\sum_{\substack{(p-1) \mid n \\
p \text { odd }}} p^{-c} & \leq \sum_{\substack{d \mid n \\
d \text { even }}}(d+1)^{-c}<\sum_{\substack{d \mid n \\
d \text { even }}} d^{-c} \leq 2^{-c} \sum_{d \mid n} d^{-c} \\
& \leq 2^{-c} \prod_{p \mid n}\left(1-p^{-c}\right)^{-1}=2^{-c} f_{c}(n) .
\end{aligned}
$$

We recall that $K_{c}=\exp \left(\sum_{p>2} \sum_{k=2}^{\infty} \frac{1}{k} p^{-k c}\right)$, which is finite for $c>\frac{1}{2}$. Thus we have Lemma 2.5 .

Proof of Lemma 2.6. From Euler's summation formula,

$$
\sum_{k \leq x} \frac{1}{k} \leq 1+\int_{1}^{x} \frac{1}{t} d t=1+\log x
$$

Using partial summation,

$$
\sum_{k \leq x} \frac{\tau(k)}{k}=\frac{1}{x} \sum_{k \leq x} \tau(k)+\int_{1}^{x} \frac{1}{t^{2}} \sum_{k \leq t} \tau(k) d t
$$

We have by the first part of the lemma

$$
\sum_{k \leq t} \tau(k)=\sum_{k \leq t} \sum_{d \mid k} 1=\sum_{d \leq t}\left[\frac{t}{d}\right] \leq \sum_{d \leq t} \frac{t}{d} \leq t(1+\log t) .
$$

Thus,

$$
\begin{aligned}
\sum_{k \leq x} \frac{\tau(k)}{k} & \leq \frac{1}{x} \cdot x(1+\log x)+\int_{1}^{x} \frac{1+\log t}{t} d t \\
& =1+\log x+\frac{1}{2} \log ^{2} x+\log x \\
& <\frac{1}{2}(\log x+2)^{2}
\end{aligned}
$$

3. The Range $x \geq 10^{300}$. In this section we shall use Theorem 2.3 to prove (1.2) and establish the estimates in Table 1 for $x \geq 10^{300}$. For the record, we make the following formal statement.

THEOREM 3.1. If $x \geq 10^{10^{5}}$, then $P(x) \leq 1 /(\log x)^{197}$.

We shall prove Theorem 3.1 by choosing

$$
L=(\log x)^{200}, \quad L_{1}=\frac{L}{(\log L)^{2}}, \quad c=0.75
$$


in Theorem 2.3. However, there is some substantial work to do since the last term in Theorem 2.2 is not in closed form. The last term in Theorem 2.2 is

$$
\frac{K_{c}}{1-c} x^{1+c} L^{2(1-c)}\left(1+\log L_{1}\right) \sum_{m \leq L^{2} L_{1}} \frac{\tau_{L_{1}}(m)}{m} \exp \left(2^{-c} f_{c}(m)\right),
$$

where

$$
\begin{gathered}
K_{c}=\exp \left(\sum_{p \text { odd }} \sum_{k=2}^{\infty} k^{-1} p^{-k c}\right), \\
f_{c}(m)=\prod_{p \mid \dot{m}}\left(1-p^{-c}\right)^{-1}
\end{gathered}
$$

and $\tau_{L_{1}}(m)$ is the number of divisors of $m$ up to $L_{1}$.

To get an upper bound for (3.2), we first get an upper bound for $K_{c}$. Let $p_{i}$ denote the $i$ th prime. We have

$$
\begin{aligned}
\sum_{p>2} \sum_{k=2}^{\infty} \frac{1}{k} p^{-k c} & \leq \frac{1}{2} \sum_{p>2} p^{-2 c}+\frac{1}{3} \sum_{p>2} p^{-3 c}\left(1+p^{-c}+p^{-2 c}+\cdots\right) \\
& =\frac{1}{2} \sum_{p>2} p^{-2 c}+\frac{1}{3} \sum_{p>2} \frac{p^{-3 c}}{1-p^{-c}}=\sum_{p>2} p^{-2 c}\left(\frac{1}{2}+\frac{1}{3\left(p^{c}-1\right)}\right) \\
& \leq \sum_{i=2}^{11} p_{i}^{-2 c}\left(\frac{1}{2}+\frac{1}{3\left(p_{i}^{c}-1\right)}\right)+\left(\frac{1}{2}+\frac{1}{3\left(37^{c}-1\right)}\right) \sum_{p \geq 37} p^{-2 c}
\end{aligned}
$$

Now,

$$
\begin{aligned}
\sum_{p \geq 37} p^{-2 c} & <\sum_{k=18}^{\infty}(2 k+1)^{-2 c}<\sum_{k=18}^{\infty} \int_{2 k}^{2 k+1} t^{-2 c} d t \\
& <\int_{36}^{37} t^{-2 c} d t+\frac{1}{2} \sum_{k=19}^{\infty}\left(\int_{2 k-1}^{2 k}+\int_{2 k}^{2 k+1}\right) t^{-2 c} d t \\
& =\int_{36}^{37} t^{-2 c} d t+\frac{1}{2} \int_{37}^{\infty} t^{-2 c} d t \\
& =\frac{1}{2 c-1}\left(36^{1-2 c}-\frac{1}{2} \cdot 37^{1-2 c}\right) .
\end{aligned}
$$

Thus, if

$$
\begin{aligned}
K_{c}^{\prime}= & \sum_{i=2}^{11} p_{i}^{-2 c}\left(\frac{1}{2}+\frac{1}{3\left(p_{i}^{c}-1\right)}\right) \\
& +\frac{1}{(2 c-1)}\left(\frac{1}{2}+\frac{1}{3\left(37^{c}-1\right)}\right)\left(36^{1-2 c}-\frac{1}{2} \cdot 37^{1-2 c}\right),
\end{aligned}
$$

then

$$
K_{c} \leq \exp \left(K_{c}^{\prime}\right)
$$

We now obtain an upper bound for $\sum_{m \leq L^{2} L_{1}} \tau_{L_{1}}(m) / m$. 
LEMMA 3.2. If $1<L_{1}<L$, then

$$
\sum_{m \leq L^{2} L_{1}} \frac{\tau_{L_{1}}(m)}{m} \leq\left(1+\log L_{1}\right)\left(1+\log L^{2} L_{1}\right) .
$$

Proof. We have

$$
\sum_{m \leq L^{2} L_{1}} \frac{\tau_{L_{1}}(m)}{m}=\frac{1}{L^{2} L_{1}} \sum_{m \leq L^{2} L_{1}} \tau_{L_{1}}(m)+\int_{1}^{L^{2} L_{1}} \frac{1}{t^{2}} \sum_{m \leq t} \tau_{L_{1}}(m) d t .
$$

Note that by Lemma 2.6

$$
\begin{aligned}
\sum_{m \leq t} \tau_{L_{1}}(m) & =\sum_{m \leq t} \sum_{\substack{d \mid m \\
d \leq L_{1}}} 1=\sum_{d \leq L_{1}}\left[\frac{t}{d}\right] \\
& \leq t \sum_{d \leq L_{1}} \frac{1}{d} \leq t\left(1+\log L_{1}\right) .
\end{aligned}
$$

Thus,

$$
\begin{aligned}
\sum_{m \leq L^{2} L_{1}} \frac{\tau_{L_{1}}(m)}{m} & \leq\left(1+\log L_{1}\right)+\int_{1}^{L^{2} L_{1}} \frac{1}{t}\left(1+\log L_{1}\right) d t \\
& =\left(1+\log L_{1}\right)\left(1+\log L^{2} L_{1}\right) .
\end{aligned}
$$

This completes the proof of Lemma 3.2.

Using Lemma 3.2, we can get an upper bound for

We define

$$
\sum_{m \leq L^{2} L_{1}} \frac{\tau_{L_{1}}(m)}{m} \exp \left(2^{-c} f_{c}(m)\right)
$$

$$
m_{i}=p_{1} p_{2} \cdots p_{i},
$$

the product of the first $i$ primes, and let $j$ be an integer such that

$$
m_{j} \leq L^{2} L_{1}<m_{j+1} .
$$

PROPOSITION 3.3. If $1<L_{1}<L$, then

$$
\begin{aligned}
& \sum_{m \leq L^{2} L_{1}} \frac{\tau_{L_{1}}(m)}{m} \exp \left(2^{-c} f_{c}(m)\right) \\
& \quad \leq\left(1+\log L_{1}\right)\left(1+\log L^{2} L_{1}\right) \exp \left(2^{-c} f_{c}\left(m_{j}\right)\right) .
\end{aligned}
$$

Proof. Suppose that $m$ has $k$ distinct prime factors $q_{1}, q_{2}, \ldots, q_{k}$ and that $p_{1}, p_{2}, \ldots, p_{k}$ are the first $k$ primes. Then

$$
f_{c}(m)=\prod_{1 \leq i \leq k}\left(1-q_{i}^{-c}\right)^{-1} \leq \prod_{1 \leq i \leq k}\left(1-p_{i}^{-c}\right)^{-1}=f_{c}\left(m_{k}\right) .
$$

Because $j$ is chosen as $p_{1} p_{2} \cdots p_{j} \leq L^{2} L_{1}<p_{1} p_{2} \cdots p_{j+1}$, clearly $j$ is the largest possible value for the number of primes in $m \leq L^{2} L_{1}$. Thus $f_{c}\left(m_{j}\right)$ is a universal upper bound for $f_{c}(m)$ for any $m \leq L^{2} L_{1}$. Thus,

$$
\begin{aligned}
\sum_{m \leq L^{2} L_{1}} \frac{\tau_{L_{1}}(m)}{m} \exp \left(2^{-c} f_{c}(m)\right) & \leq \exp \left(2^{-c} f_{c}\left(m_{j}\right)\right) \sum_{m \leq L^{2} L_{1}} \frac{\tau_{L_{1}}(m)}{m} \\
& \leq \exp \left(2^{-c} f_{c}\left(m_{j}\right)\right)\left(1+\log L_{1}\right)\left(1+\log L^{2} L_{1}\right)
\end{aligned}
$$

by Lemma 3.2 . 
Now we prove Theorem 3.1. Assume $x \geq 10^{10^{5}}$ and $L, L_{1}, c$ are as given in (3.1). From Theorem 2.3 and Proposition 3.3, we have

$$
\begin{aligned}
P(x) \leq & \frac{\sum_{n \leq x, n \text { odd, composite }} F(n)}{\sum_{2<p \leq x}(p-3)} \\
\leq 2(2+\log x)\left(\frac{(\log L)^{2}}{4 L}+\frac{\log L}{L}+\frac{(\log L)^{2}}{L}\right. & \\
& \left.\quad+\frac{3 K_{c}}{1-c} \cdot \frac{L^{2(1-c)}}{x^{1-c}}(\log L)^{3} \exp \left(2^{-c} f_{c}\left(m_{j}\right)\right)\right) \\
=2(2+\log x)( & \frac{5(\log L)^{2}+4 \log L}{4 L} \\
& \left.\quad+\frac{3 K_{c}}{1-c} \cdot \frac{L^{2(1-c)}}{x^{1-c}}(\log L)^{3} \exp \left(2^{-c} f_{c}\left(m_{j}\right)\right)\right) .
\end{aligned}
$$

We shall be interested in getting an upper bound for $2^{-c} f_{c}\left(m_{j}\right)$. We have

$$
2^{-c} f_{c}\left(m_{j}\right)=2^{-c} \prod_{p \mid m_{j}}\left(1-p^{-c}\right)^{-1}=\frac{1}{2^{c}-1} \prod_{i=2}^{j}\left(1-p_{i}^{-c}\right)^{-1} .
$$

Now,

$$
\log \prod_{i=2}^{j}\left(1-p_{i}^{-c}\right)^{-1}=-\sum_{i=2}^{j} \log \left(1-p_{i}^{-c}\right)=\sum_{i=2}^{j} p_{i}^{-c}+\sum_{i=2}^{j} \sum_{k=2}^{\infty} \frac{1}{k} p_{i}^{-k c} .
$$

Hence,

$$
\prod_{i=2}^{j}\left(1-p_{i}^{-c}\right)^{-1} \leq K_{c} \exp \left(\sum_{i=2}^{j} p_{i}^{-c}\right)
$$

Putting (3.8) in (3.7), we have

$$
2^{-c} f_{c}\left(m_{j}\right) \leq \frac{K_{c}}{2^{c}-1} \exp \left(\sum_{i=2}^{j} p_{i}^{-c}\right) .
$$

Now for $j>16$,

$$
\sum_{i=2}^{j} p_{i}^{-c}=\sum_{i=2}^{16} p_{i}^{-c}+\sum_{i=17}^{j} p_{i}^{-c}
$$

Using partial summation for $\sum_{i=17}^{j} p_{i}^{-c}$, we have

$$
\sum_{i=17}^{j} p_{i}^{-c}=\sum_{59 \leq p \leq p_{j}} p^{-c}=j p_{j}^{-c}-16 \cdot 59^{-c}+c \int_{59}^{p_{j}} t^{-c-1} \pi(t) d t .
$$

We use the following upper bound for $\pi(t)$ :

$$
\pi(t) \leq \frac{t}{\log t}\left(1+\frac{3}{2 \log t}\right) \quad \text { for } t>1
$$


which is (3.2) in [5, p. 69]. Thus, for $S=\int_{59}^{p_{j}} t^{-c-1} \pi(t) d t$, we have

$$
\begin{aligned}
S & \leq \int_{59}^{p_{j}} \frac{t^{-c}}{\log t}\left(1+\frac{3}{2 \log t}\right) d t \\
& \left.=\frac{1}{1-c} \cdot \frac{t^{1-c}}{\log t}\right]_{59}^{p_{j}}+\left(\frac{3}{2}+\frac{1}{1-c}\right) \int_{59}^{p_{j}} \frac{t^{-c}}{\log ^{2} t} d t .
\end{aligned}
$$

Let $I=\int_{59}^{p_{j}}\left(t^{-c} / \log ^{2} t\right) d t$. Assuming $p_{j}>3481=59^{2}$, we have

$$
\begin{aligned}
I= & \int_{59}^{\sqrt{p_{j}}} \frac{t^{-c}}{\log ^{2} t} d t+\int_{\sqrt{p_{j}}}^{p_{j}} \frac{t^{-c}}{\log ^{2} t} d t \\
\leq & \left.\frac{1}{\log ^{2} 59} \cdot \frac{1}{1-c} \cdot t^{1-c}\right]_{59}^{\sqrt{p_{j}}} \\
& \left.+\frac{1}{\log ^{2} \sqrt{p_{j}}} \cdot \frac{1}{1-c} \cdot t^{1-c}\right]_{\sqrt{p_{j}}}^{p_{j}} .
\end{aligned}
$$

Thus,

$$
\begin{aligned}
& S \leq \frac{1}{1-c} \frac{p_{j}^{1-c}}{\log p_{j}}-\frac{1}{1-c} \frac{59^{1-c}}{\log 59} \\
&+\left(\frac{3}{2}+\frac{1}{1-c}\right)\left(\frac{1}{(1-c) \log ^{2} 59} p_{j}^{(1-c) / 2}-\frac{1}{(1-c) \log ^{2} 59} 59^{1-c}\right. \\
&\left.\quad+\frac{4}{1-c} \cdot \frac{p_{j}^{1-c}}{\log ^{2} p_{j}}-\frac{4}{1-c} \frac{p_{j}^{(1-c) / 2}}{\log ^{2} p_{j}}\right) .
\end{aligned}
$$

Now by (3.12),

$$
j=\pi\left(p_{j}\right)<\frac{p_{j}}{\log p_{j}}\left(1+\frac{3}{2 \log p_{j}}\right) .
$$

Assembling (3.10), (3.11), (3.14), (3.15), and simplifying with $c=0.75$, we have

$$
\sum_{i=2}^{j} p_{i}^{-0.75} \leq 4 \cdot \frac{p_{j}^{1 / 4}}{\log p_{j}}+67.5 \frac{p_{j}^{1 / 4}}{\log ^{2} p_{j}}+p_{j}^{1 / 8}-66 \cdot \frac{p_{j}^{1 / 8}}{\log ^{2} p_{j}}-3.7 .
$$

From (3.5) and Theorems 9 and 10 in [5, p. 71], we have

$$
p_{j} \leq 1.04 \log L^{2} L_{1} \leq 3.12 \log L \text { for } \log L^{2} L_{1}>2703 .
$$

Putting this in (3.16) and taking $L=(\log x)^{200}$, we get

$$
\sum_{i=2}^{j} p_{i}^{-0.75} \leq 4.8(\log \log x)^{1 / 4} \text { for } x \geq 10^{10^{5}}
$$

From (3.3) with $c=0.75$, we have $K_{c}<e^{0.4}$. Thus, from the above and (3.9), we have

$$
\begin{aligned}
\exp \left(2^{-c} f_{c}\left(m_{j}\right)\right) & \leq \exp \left(\frac{K_{c}}{2^{c}-1} \exp \left(\sum_{i=2}^{j} p_{i}^{-0.75}\right)\right) \\
& <\exp \left(2.2 \exp \left(4.8(\log \log x)^{1 / 4}\right)\right)<x^{0.1}
\end{aligned}
$$

for $x \geq 10^{10^{5}}$. 
We put (3.17) in (3.6), getting

$$
\begin{aligned}
P(x) \leq & 2(2+\log x) \\
& \cdot\left(\frac{5(\log L)^{2}+4 \log L}{4 L}+18.0 \frac{L^{1 / 2}}{x^{0.15}}(\log L)^{3}\right)
\end{aligned}
$$

for $x \geq 10^{10^{5}}$, where $L=(\log x)^{200}$. Since $x^{0.15}>(\log x)^{1200}$ for all $x \geq 10^{10^{5}}$, it is easy to check that (3.18) implies $P(x) \leq 1 /(\log x)^{197}$ for all $x \geq 10^{10^{5}}$, which was to be proved.

To establish the estimates in Table 1 for $x \geq 10^{300}$, we use the same values of the parameters $L_{1}, c$ in Theorem 2.3 as given in (3.1) and then we choose $L$ optimally. We also use Proposition 3.3 for the sum in the last term in Theorem 2.2 , to obtain (3.6). The principal difference between the range $x \geq 10^{10^{5}}$ and $10^{300} \leq x \leq 10^{10^{5}}$ is that instead of using (3.7)-(3.17) to estimate $\exp \left(2^{-c} f_{c}\left(m_{j}\right)\right)$, we directly compute this quantity, which is not too hard to do when given a finite value of $x$ that is not too large.

4. A Refinement of the Basic Method. To get good results for smaller values of $x$, we shall use a more elaborate version of Theorem 2.2 and Proposition 3.3 .

THEOREM 4.1. Suppose $c, L_{1}, L, L_{2}$ and $M$ are arbitrary real numbers satisfying $\frac{1}{2}<c<1,10<L_{1}<L<L_{2}<M / 2, L^{3 / 2} \leq 10 M$. Then for any $x>L^{2}$, we have

$$
\begin{aligned}
\sum_{\substack{n \leq x, n \text { odd, } \\
\text { composite }}} F(n) \leq & \frac{x^{2}}{4 L_{1}}+\frac{50}{99} \frac{x^{2}}{L_{2}-1}\left(\frac{L_{1}}{L_{2}-1}+1\right)\left(2+\log L_{1}\right)^{2} \\
& +x L_{2}^{2}\left(2+\frac{\log x}{\log 10}\right)\left(1+\log L_{1}\right)+\frac{100}{99} \frac{x^{2}}{M}\left(1+\log L_{1}\right)^{2} \\
& +\frac{125}{3564} \frac{x^{2}\left(1+\log L_{2}\right)^{2}}{M-2 L_{2}}\left(4+\log L_{1}\right)^{4}+\frac{50}{99} \frac{x^{2}}{L}\left(1+\log L_{1}\right) \\
& +\frac{100 K_{c}}{(1-c)\left(10^{1+c}-1\right)} x^{1+c} M^{1-c}\left(1+\log L_{1}\right) \\
& \cdot \sum_{m \leq L^{2} L_{1}} \frac{\tau_{L_{1}}(m)}{m} \exp \left(2^{-c} f_{c}(m)\right),
\end{aligned}
$$

where $K_{c}, f_{c}(m)$ and $\tau_{L_{1}}(m)$ are defined in Theorem 2.2 .

Proof. Although the assertion appears to be considerably more complicated, the proof of Theorem 4.1 follows fairly directly from the same methods used to prove Theorem 2.2. By the same argument that establishes (2.6), we have

$$
\sum_{\substack{x / 10<n \leq x \\ n \text { odd, composite }}} F(n) \leq x \sum_{k \leq L_{1}} \frac{C_{k}(x)-C_{k}(x / 10)}{k}+\frac{1}{L_{1}} \sum_{\substack{x / 10<n \leq x \\ n \text { odd }}}(n-2) .
$$


To obtain the theorem, we add (4.1) at $x, x / 10, \ldots, x / 10^{u}$, where $x / 10^{u}>L^{2}>$ $x / 10^{u+1}$. Thus it shall be sufficient to prove that

$$
\begin{aligned}
\sum_{k \leq L_{1}} & \frac{C_{k}(x)-C_{k}(x / 10)}{k} \\
\leq & \frac{1}{2} \cdot \frac{x}{L_{2}-1}\left(\frac{L_{1}}{L_{2}-1}\right)\left(2+\log L_{1}\right)^{2}+\frac{x}{M}\left(1+\log L_{1}\right)^{2} \\
& +\frac{5}{144} \frac{x\left(1+\log L_{2}\right)^{2}}{M-2 L_{2}}\left(4+\log L_{1}\right)^{4}+\frac{1}{2} \frac{x}{L}\left(1+\log L_{1}\right) \\
& +L_{2}^{2}\left(1+\log L_{1}\right) \\
& +\frac{10^{1-c} K_{c}}{1-c} x^{c} M^{1-c}\left(1+\log L_{1}\right) \sum_{m \leq L^{2} L_{1}} \frac{\tau_{L_{1}}(m)}{m} \exp \left(2^{-c} f_{c}(m)\right) .
\end{aligned}
$$

The expression $C_{k}(x)-C_{k}(x / 10)$ is the cardinality of the set of odd, composite integers $n$ with $x / 10<n \leq x$ and $F(n)=\phi(n) / k$. We let $B_{k, 1}(x), B_{k, 2}(x), B_{k, 3}(x)$ denote the number of such $n$ that satisfy, respectively,

(1) $n$ is divisible by some prime $p>L_{2}$,

(2) $n$ has a divisor $p q>M$, where $q \leq p$ are prime, but $n$ is not counted by $(1)$

(3) $n$ is not counted by (1) or (2).

Thus, $C_{k}(x)-C_{k}(x / 10)=B_{k, 1}(x)+B_{k, 2}(x)+B_{k, 3}(x)$.

From the argument which gives (2.12) we have immediately that

$$
B_{k, 1}(x) \leq \frac{x}{L_{2}-1}\left(\frac{L_{1}}{L_{2}-1}\right) \tau(k),
$$

so that from Lemma 2.6 , we have

$$
\sum_{k \leq L_{1}} \frac{B_{k, 1}(x)}{k} \leq \frac{1}{2} \cdot \frac{x}{L_{2}-1}\left(\frac{L_{1}}{L_{2}-1}\right)\left(2+\log L_{1}\right)^{2} .
$$

To analyze $B_{k, 2}(x)$, we consider separately the case $q<p$ and $q=p$. The contribution to $B_{k, 2}(x)$ from the case $q<p$ is, by (2.10), at most

$$
\begin{aligned}
& \sum_{\substack{q<p \leq L_{2} \\
p q>M}} 1+\frac{x(k,[p-1, q-1])}{p q[p-1, q-1]} \\
& \leq \frac{1}{2} L_{2}^{2}+\frac{1}{2} x \sum_{\substack{q, p \leq L_{2} \\
p q>M \\
p \neq q}} \frac{(k,[p-1, q-1])}{p q[p-1, q-1]} .
\end{aligned}
$$

For this last sum, we write $p-1=m d, q-1=n d$, where $(m, n)=1$. Since $p q>M$ and $p, q \leq L_{2}$ imply $(p-1)(q-1)>M-2 L_{2}:=M^{\prime}$, we have

$$
\sum_{\substack{p, q \leq L_{2} \\
p q>M \\
p \neq q}} \frac{(k,[p-1, q-1])}{p q[p-1, q-1]} \leq \sum_{\substack { d \leq L_{2} \\
\begin{subarray}{c}{m, n \leq L_{2} / d \\
m n>M^{\prime} / d^{2} \\
(m, n)=1{ d \leq L _ { 2 } \\
\begin{subarray} { c } { m , n \leq L _ { 2 } / d \\
m n > M ^ { \prime } / d ^ { 2 } \\
( m , n ) = 1 } }\end{subarray}} \frac{(k, m n d)}{m^{2} n^{2} d^{3}} .
$$


If we let $u_{1}=(k, m), u_{2}=(k, n)$, then the condition $(m, n)=1$ implies $u_{1} u_{2} \mid k$. Let $u_{3}=\left(k / u_{1} u_{2}, d\right)$. Thus, $(k, m n d)=u_{1} u_{2} u_{3}$. Let $u_{4}$ be such that $k=u_{1} u_{2} u_{3} u_{4}$, and let $\mu, \nu, \delta$ be such that $m=u_{1} \mu, n=u_{2} \nu, d=u_{3} \delta$. Thus, from (4.5),

$$
\begin{aligned}
& \sum_{\substack{p, q \leq L_{2} \\
p q>M \\
p \neq q}} \frac{(k,[p-1, q-1])}{p q[p-1, q-1]} \\
& \quad \leq \sum_{\substack{u_{1} u_{2} u_{3} u_{4}=k \\
\left(u_{1}, u_{2}\right)=1}} \sum_{\substack{\delta \leq L_{2} / u_{3} \\
\nu \leq L_{2} / u_{2}}} \sum_{\mu>M^{\prime} /\left(\nu \delta^{2} u_{1} u_{2} u_{3}^{2}\right)} \frac{1}{\mu^{2} \nu^{2} \delta^{3} u_{1} u_{2} u_{3}^{2}} \\
& \quad<\frac{5}{3 M^{\prime}} \sum_{u_{1} u_{2} u_{3} u_{4}=k} \sum_{\substack{\delta \leq L_{2} / u_{3} \\
\nu \leq L_{2} / u_{2}}} \frac{1}{\nu \delta} \\
& \quad \leq \frac{5}{3 M^{\prime}}\left(1+\log L_{2}\right)^{2} \sum_{u_{1} u_{2} u_{3} u_{4}=k} 1,
\end{aligned}
$$

where we used the inequality

$$
\sum_{\mu>y} \frac{1}{\mu^{2}}<\frac{5}{3 y} \quad \text { for } y>0 .
$$

The proof of (4.7) follows from the facts that

$$
\begin{aligned}
& \sum_{\mu>y} \frac{1}{\mu^{2}}=\sum_{\mu=1}^{\infty} \frac{1}{\mu^{2}}=\frac{\pi^{2}}{6}<\frac{5}{3}<\frac{5}{3 y} \quad \text { for } 0<y<1 \\
& \sum_{\mu>y} \frac{1}{\mu^{2}} \leq \sum_{\mu=2}^{\infty} \frac{1}{\mu^{2}}<\frac{2}{3} \leq \frac{5}{3 y} \quad \text { for } 1 \leq y \leq 2.5 \\
& \sum_{\mu>y} \frac{1}{\mu^{2}}<\int_{y-1}^{\infty} \frac{1}{t^{2}} d t=\frac{1}{y-1}<\frac{5}{3 y} \quad \text { for } y>2.5
\end{aligned}
$$

Putting (4.6) into (4.4), we have

$$
\begin{aligned}
\sum_{k \leq L_{1}} & \frac{1}{k} \sum_{\substack{q<p \leq L_{2} \\
p q>M}} 1+\frac{x(k,[p-1, q-1])}{p q[p-1, q-1]} \\
& \leq \frac{1}{2} L_{2}^{2}\left(1+\log L_{1}\right)+\frac{5 x}{6 M^{\prime}}\left(1+\log L_{2}\right)^{2} \sum_{k \leq L_{1}} \frac{\tau_{(4)}(k)}{k}
\end{aligned}
$$

where $\tau_{(i)}(k)$ is the number of ordered factorizations of $k$ into $i$ positive factors.

It is not hard to prove by induction on $i$ that

$$
\sum_{k \leq y} \frac{\tau_{(i)}(k)}{k} \leq \frac{1}{i !}(i+\log y)^{i}
$$

for any natural number $i$ and for any $y \geq 1$; in fact, Lemma 2.6 gives the cases $i=1,2$. We shall use (4.9) with $i=4$ in a moment.

We now consider the contribution to $B_{k, 2}(x)$ when $q=p$. If $p^{2} \mid n$ and $F(n)=$ $\phi(n) / k$, we have from (2.9) that

$$
n \equiv 0\left(\bmod p^{2}\right), \quad k(n-1) \equiv 0(\bmod p(p-1)) .
$$


Thus, $p \mid k$, and the number of such $n \leq x$ is at most

$$
1+\left[\frac{x(k, p-1)}{p^{2}(p-1)}\right] \leq 1+\frac{x}{p^{2}}<1+\frac{x}{M}
$$

if $p^{2}>M$. Since $k \leq L_{1}$, the number of primes $p \mid k$ with $p>M^{1 / 2}$ is at most $\left(\log L_{1}\right) / \log \left(M^{1 / 2}\right)<\log L_{1}$. Thus, the contribution to $B_{k, 2}(x)$ when $q=p$ is at most $(1+x / M) \log L_{1}$.

Using this result together with (4.8) and (4.9) gives

$$
\begin{aligned}
\sum_{k \leq L_{1}} \frac{B_{k, 2}(x)}{k} \leq & L_{2}^{2}\left(1+\log L_{1}\right)+\frac{x}{M}\left(1+\log L_{1}\right)^{2} \\
& +\frac{5 x}{144 M^{\prime}}\left(1+\log L_{2}\right)^{2}\left(4+\log L_{1}\right)^{4}
\end{aligned}
$$

We now turn our attention to $B_{k, 3}(x)$, the number of odd, composite $n$ with $x / 10<n \leq x, p \leq L_{2}$ for every prime $p \mid n$, and $p q \leq M$ for all primes $p, q$ with $p q \mid n$. Factor such a number $n$ as $q_{1} q_{2} \cdots q_{t}$, where $q_{1} \geq q_{2} \geq \cdots \geq q_{t}$ are primes. Then, $q_{1} \leq L_{2}$ and $q_{1} q_{2} \leq M$. Note that

$$
\frac{n}{q_{1} q_{2}} \geq \frac{n}{M}>\frac{x}{10 M}
$$

Suppose $n / q_{1} q_{2}>x / L$. Then, $q_{1} q_{2} \leq L$, so that $q_{2} \leq L^{1 / 2}$. Thus, $n$ has a divisor $d$ with $x / L^{3 / 2}<d \leq x / L$. But $x / L^{3 / 2} \geq x /(10 M)$, so that in either case, $n$ has a divisor $d$ satisfying

$$
\frac{x}{10 M}<d \leq \frac{x}{L}
$$

We next repeat the calculations $(2.15)-(2.18)$, but with $\sum^{\prime}$ now representing a sum over odd $d$ satisfying (4.11). Thus,

$$
B_{k, 3}(x) \leq \frac{x}{2 L}+\frac{K_{c}}{1-c} x^{c}(10 M)^{1-c} \sum_{u \mid k} \sum_{m \leq L^{2}} \frac{1}{m} \exp \left(2^{-c} f_{c}(m u)\right)
$$

and so, as in Section 2, we get

$$
\begin{aligned}
& \sum_{k \leq L_{1}} \frac{B_{k, 3}(x)}{k} \leq \frac{x}{2 L}\left(1+\log L_{1}\right)+\frac{K_{c}}{1-c} x^{c}(10 M)^{1-c}\left(1+\log L_{1}\right) \\
& \cdot \sum_{m \leq L^{2} L_{1}} \frac{\tau_{L_{1}}(m)}{m} \exp \left(2^{-c} f_{c}(m)\right) .
\end{aligned}
$$

Our proof is now complete, since adding (4.3), (4.10) and (4.12) gives (4.2), which, as we have seen, is sufficient for the proof of the theorem.

We shall also wish to use a sharper result than Proposition 3.3. Let

$$
2 \leq l<L_{1}, \quad \alpha_{l}=\left(1-l^{-c}\right)^{-\log \left(L^{2} L_{1}\right) / \log l},
$$

where $\alpha_{l}$ depends on the choice of $c, L, L_{1}, l$. 
Proposition 4.2. If $\frac{1}{2}<c<1,1<L_{1}<L$ and $l, \alpha_{l}$ are given by (4.13), we have

$$
\begin{aligned}
& \sum_{m \leq L^{2} L_{1}} \frac{\tau_{L_{1}}(m)}{m} \exp \left(2^{-c} f_{c}(m)\right) \\
& \quad \leq\left(1+\log L_{1}\right) \sum_{i=0}^{j} \frac{1}{i !}\left(2 \sum_{p \leq l} \frac{1}{p}\right)^{i} \exp \left(2^{-c} \alpha_{l} f_{c}\left(m_{i}\right)\right)\left(1+\log \frac{L^{2} L_{1}}{m_{i}}\right)
\end{aligned}
$$

where $m_{i}$ and $j$ are defined in (3.4) and (3.5).

To prove Proposition 4.2, we state a result that will be useful. We first make a definition as follows. Let $S(l, k)=\sum \frac{1}{u}$, where $u$ runs over the squarefree integers that are the product of $k$ distinct primes up to $l$.

LEMMA 4.3. For any nonnegative integer $k$ and any $l \geq 2$, we have

$$
S(l, k) \leq \frac{1}{k !}\left(\sum_{p \leq l} \frac{1}{p}\right)^{k} .
$$

Proof. This elementary result follows by expanding the right side of this inequality with the multinomial theorem.

We now prove Proposition 4.2.

Proof of Proposition 4.2. To estimate $f_{c}(m)$, we ask how many primes $p>l$ can divide $m$. The number of such primes is at most $\log \left(L^{2} L_{1}\right) / \log l$. Thus,

$$
\begin{aligned}
f_{c}(m) & =\prod_{p \mid n}\left(1-p^{-c}\right)^{-1}=\prod_{\substack{p \mid m \\
p>l}}\left(1-p^{-c}\right)^{-1} \cdot \prod_{\substack{p \mid m \\
p \leq l}}\left(1-p^{-c}\right)^{-1} \\
& \leq\left(1-l^{-c}\right)^{-\log \left(L^{2} L_{1}\right) / \log l} \cdot \prod_{\substack{p \mid m \\
p \leq l}}\left(1-p^{-c}\right)^{-1} \leq \alpha_{l} f_{c}\left(m_{i}\right)
\end{aligned}
$$

if $m$ has exactly $i$ distinct primes up to $l$. In general, let $\omega_{l}(m)$ be the number of distinct prime factors of $m$ at most $l$ and $\omega(m)$ be the number of distinct primes in $m$.

Now,

$$
\sum_{m \leq L^{2} L_{1}} \frac{\tau_{L_{1}}(m)}{m} \exp \left(2^{-c} f_{c}(m)\right) \leq \sum_{i=0}^{j} \sum_{\substack{\omega_{l}(m)=i \\ m \leq L^{2} L_{1}}} \frac{\tau_{L_{1}}(m)}{m} \exp \left(2^{-c} f_{c}(m)\right) .
$$

For the inner sum, we have

$$
\begin{gathered}
\sum_{\substack{\omega_{l}(m)=i \\
m \leq L^{2} L_{1}}} \frac{\tau_{L_{1}}(m)}{m} \exp \left(2^{-c} f_{c}(m)\right) \leq \exp \left(2^{-c} \alpha_{l} f_{c}\left(m_{i}\right)\right) \sum_{\begin{array}{c}
\omega_{l}(m)=i \\
m \leq L^{2} L_{1}
\end{array}} \frac{\tau_{L_{1}}(m)}{m} \\
\leq \exp \left(2^{-c} \alpha_{l} f_{c}\left(m_{i}\right)\right) \sum_{\substack{\omega(u)=\omega_{l}(u)=i \\
u \text { squarefree }}} \sum_{t \leq L^{2} L_{1} / m_{i}} \frac{\tau_{L_{1}}(u t)}{u t}
\end{gathered}
$$


since any $m \leq L^{2} L_{1}$ with $\omega_{l}(m)=i$ may be factored as $u t$, where $u$ is the product of $i$ distinct primes up to $l$ and $t$ is an integer at most $L^{2} L_{1} / u \leq L^{2} L_{1} / m_{i}$. Using

$$
\tau_{L_{1}}(u t) \leq \tau_{L_{1}}(u) \tau_{L_{1}}(t) \leq \tau(u) \tau_{L_{1}}(t)=2^{i} \tau_{L_{1}}(t)
$$

we have

$$
\begin{aligned}
& \sum_{\substack{\omega_{l}(m)=i \\
m \leq L^{2} L_{1}}} \frac{\tau_{L_{1}}(m)}{m} \exp \left(2^{-c} f_{c}(m)\right) \\
& \leq \exp \left(2^{-c} \alpha_{l} f_{c}\left(m_{i}\right)\right) \sum_{\substack{\omega(u)=\omega_{l}(u)=i \\
u \text { squarefree }}} \frac{2^{i}}{u} \sum_{t \leq L^{2} L_{1} / m_{i}} \frac{\tau_{L_{1}}(t)}{t} \\
& \leq \exp \left(2^{-c} \alpha_{l} f_{c}\left(m_{i}\right)\right) \frac{1}{i !}\left(2 \sum_{p \leq l} \frac{1}{p}\right)^{i}\left(1+\log L_{1}\right)\left(1+\log \frac{L^{2} L_{1}}{m_{i}}\right)
\end{aligned}
$$

by Lemmas 3.2 and 4.3. Therefore, taking the summation of (4.14) from $i=0$ to $j$ completes the proof.

Putting together Theorems 2.1 and 4.1 and Proposition 4.2, we have the following result.

THEOREM 4.4. If $\frac{1}{2}<c<1,10<L_{1}<L<L_{2}<M / 2, L^{3 / 2} \leq 10 M$, and $x>L^{2}$, then

$$
\left.\begin{array}{rl}
P(x) \leq 2(2+\log x)\{ & \frac{1}{4 L_{1}}+\frac{50}{99}\left(\frac{L_{1}}{L_{2}-1}+1\right) \frac{\left(2+\log L_{1}\right)^{2}}{L_{2}-1} \\
& +\frac{1}{x} L_{2}^{2}\left(2+\frac{\log x}{\log 10}\right)\left(1+\log L_{1}\right)+\frac{100}{99} \cdot \frac{\left(1+\log L_{1}\right)^{2}}{M} \\
& +\frac{125}{3564} \frac{\left(1+\log L_{2}\right)^{2}}{M-2 L_{2}}\left(4+\log L_{1}\right)^{4}+\frac{50}{99} \cdot \frac{1+\log L_{1}}{L} \\
& +\frac{K_{c}}{(1-c)\left(10^{1+c}-1\right)}\left(\frac{M}{x}\right)^{1-c}\left(1+\log L_{1}\right)^{2} \\
& \cdot \sum_{i=0}^{j} \frac{1}{i !}\left(2 \sum_{p \leq l} \frac{1}{p}\right)^{i}\left(1+\log \frac{L^{2} L_{1}}{m_{i}}\right) \exp \left(2^{-c} \alpha_{l} f_{c}\left(m_{i}\right)\right)
\end{array}\right\}
$$

where $l, \alpha_{l}$ are given by (4.13), $m_{i}$ is defined in (3.4) and $j$ is defined in (3.5).

While admittedly looking complicated, Theorem 4.4 can be readily used to get explicit upper bounds for $P(x)$ for various values of $x$. The art is to choose the many free parameters optimally. Of the seven terms in the brackets, it is clear that some dominate others. For example, the fourth term is small compared to the fifth term. We choose the parameters so that the first four terms are the least important and the seventh is the most important. We feel these choices are close to the optimal ones. Our results are recorded in Table 2 and summarized in Table 1 in the Introduction. An asterisk in the $M$ column signifies that $M$ was chosen as $L^{3 / 2} / 10$. 


\section{TABLE 2}

$\begin{array}{cccccccc}x & L & L_{1} & L_{2} & M & l & c & \begin{array}{c}\text { Upper Bound } \\ \text { for } P(x)\end{array} \\ 1.0 \mathrm{E}+60 & 3.6 \mathrm{E}+5 & 5.4 \mathrm{E}+3 & 2.0 \mathrm{E}+6 & 6.2 \mathrm{E}+9 & 350 & 0.7125 & 7.16 \mathrm{E}-2 \\ 1.0 \mathrm{E}+70 & 1.1 \mathrm{E}+7 & 1.7 \mathrm{E}+5 & 1.2 \mathrm{E}+8 & 5.9 \mathrm{E}+11 & 600 & 0.7125 & 2.87 \mathrm{E}-3 \\ 1.0 \mathrm{E}+80 & 7.2 \mathrm{E}+8 & 1.1 \mathrm{E}+7 & 1.4 \mathrm{E}+10 & 9.9 \mathrm{E}+13 & 850 & 0.7125 & 8.46 \mathrm{E}-5 \\ 1.0 \mathrm{E}+90 & 4.5 \mathrm{E}+10 & 7.0 \mathrm{E}+8 & 1.4 \mathrm{E}+12 & 1.4 \mathrm{E}+16 & 1400 & 0.7100 & 1.70 \mathrm{E}-6 \\ 1.0 \mathrm{E}+100 & 3.3 \mathrm{E}+12 & 5.5 \mathrm{E}+10 & 1.7 \mathrm{E}+14 & 2.5 \mathrm{E}+18 & 1850 & 0.7100 & 2.77 \mathrm{E}-8 \\ 1.0 \mathrm{E}+110 & 2.6 \mathrm{E}+14 & 5.3 \mathrm{E}+12 & 2.3 \mathrm{E}+16 & 4.3 \mathrm{E}+20 & 1850 & 0.7100 & 4.03 \mathrm{E}-10 \\ 1.0 \mathrm{E}+120 & 1.2 \mathrm{E}+16 & 4.4 \mathrm{E}+14 & 2.5 \mathrm{E}+18 & * 1.3 \mathrm{E}+23 & 2350 & 0.7075 & 5.28 \mathrm{E}-12 \\ 1.0 \mathrm{E}+130 & 6.7 \mathrm{E}+17 & 5.6 \mathrm{E}+16 & 4.2 \mathrm{E}+20 & * 5.5 \mathrm{E}+25 & 2590 & 0.7075 & 7.54 \mathrm{E}-14 \\ 1.0 \mathrm{E}+140 & 6.8 \mathrm{E}+19 & 5.7 \mathrm{E}+18 & 5.3 \mathrm{E}+22 & * 5.6 \mathrm{E}+28 & 2800 & 0.7075 & 1.08 \mathrm{E}-15 \\ 1.0 \mathrm{E}+150 & 2.7 \mathrm{E}+21 & 2.2 \mathrm{E}+20 & 2.4 \mathrm{E}+24 & * 1.4 \mathrm{E}+31 & 3250 & 0.7075 & 1.49 \mathrm{E}-17 \\ 1.0 \mathrm{E}+160 & 4.2 \mathrm{E}+23 & 3.8 \mathrm{E}+22 & 5.1 \mathrm{E}+26 & * 2.7 \mathrm{E}+34 & 4900 & 0.7050 & 1.81 \mathrm{E}-19 \\ 1.0 \mathrm{E}+170 & 3.9 \mathrm{E}+25 & 3.5 \mathrm{E}+24 & 5.6 \mathrm{E}+28 & * 2.4 \mathrm{E}+37 & 6300 & 0.7025 & 2.27 \mathrm{E}-21 \\ 1.0 \mathrm{E}+180 & 3.7 \mathrm{E}+27 & 3.3 \mathrm{E}+26 & 6.2 \mathrm{E}+30 & * 2.3 \mathrm{E}+40 & 8000 & 0.7025 & 2.76 \mathrm{E}-23 \\ 1.0 \mathrm{E}+190 & 2.9 \mathrm{E}+29 & 2.4 \mathrm{E}+28 & 5.4 \mathrm{E}+32 & * 1.6 \mathrm{E}+43 & 9300 & 0.7025 & 3.26 \mathrm{E}-25 \\ 1.0 \mathrm{E}+200 & 2.7 \mathrm{E}+31 & 3.3 \mathrm{E}+30 & 8.2 \mathrm{E}+34 & * 1.4 \mathrm{E}+46 & 12000 & 0.7025 & 3.85 \mathrm{E}-27\end{array}$

Department of Computer Science

University of South Carolina

Columbia, South Carolina 29208

Department of Mathematics

University of Georgia

Athens, Georgia 30602

1. P. Beauchemin, G. Brassard, C. Crépeau, C. Goutier \& C. Pomerance, "The generation of random numbers that are probably prime," J. Cryptology, v. 1, 1988, pp. 53-64.

2. P. ERDÖS \& C. POMERANCE, "On the number of false witnesses for a composite number," Math. Comp., v. 46, 1986, pp. 259-279.

3. L. MONIER, "Evaluation and comparison of two efficient probabilistic primality testing algorithms," Theoret. Comput. Sci., v. 12, 1980, pp. 97-108.

4. M. O. RABIN, "Probabilistic algorithm for testing primality," J. Number Theory, v. 12, 1980, pp. 128-138.

5. J. B. Rosser \& L. SCHOENFELD, "Approximate formulas for some functions of prime numbers," Illinois J. Math., v. 6, 1962, pp. 64-94. 